\title{
A Necessary and Sufficient Condition for the Existence of Multisolitons in a Self-Dual Gauged Sigma Model
}

\author{
Yisong Yang ${ }^{\star}$
}

Department of Applied Mathematics and Physics, Polytechnic University, Brooklyn, New York 11201, USA

Received: 20 September 1995/ Accepted: 29 March 1996

\begin{abstract}
This paper presents a resolution of the gauged $O(3)$ sigma model proposed by B.J. Schroers in which the matter field $\phi$ maps $\mathbf{R}^{2}$ into $S^{2}$ while the vector gauge potential gives rise to a magnetic field. It is shown that for each natural number $N$ there are solutions to saturate the classical energy lower bound $E \geqq 4 \pi N$ for the field configurations in the topological family $\operatorname{deg}(\phi)=N$ if and only if $N \neq 1$. Furthermore the solutions obtained depend on at least $4 N-3$ continuous parameters, the associated magnetic flux can assume its value in an open interval, and the decay rates of the field strengths may be specified in a suitable range. These solutions are multisolitons represented by $N$ prescribed lumps of the magnetic field, simulating $N$ identical particles in equilibrium, and are governed by a nonlinear elliptic equation with both vortex and anti-vortex source terms.
\end{abstract}

\section{Introduction and Main Results}

In this paper we are interested in static solutions of a gauged $O(3)$ sigma model which originates from the classical planar ferromagnet model defined by the energy functional

$$
E(\phi)=\frac{1}{2} \int\left(\partial_{1} \phi\right)^{2}+\left(\partial_{2} \phi\right)^{2},
$$

where the spin vector $\phi=\left(\phi_{1}, \phi_{2}, \phi_{3}\right)$ maps $\mathbf{R}^{2}$ into $S^{2}$, namely, $\phi_{1}^{2}+\phi_{2}^{2}+\phi_{3}^{2}=1$, and the integral is taken over the full $\mathbf{R}^{2}$ under the Lebesgue measure $d^{2} x$ (unless otherwise stated). Finite energy condition implies that $\phi$ goes to a constant unit vector, say $\phi_{\infty}$, at infinity, which makes $\phi$ a continuous map from $S^{2}$ to $S^{2}$ so that the Hopf degree, $\operatorname{deg}(\phi)$, is well defined. In fact the work of Belavin and Polyakov $[1,2]$ establishes that the energy (1) has the topological lower bound

$$
E(\phi) \geqq 4 \pi|\operatorname{deg}(\phi)|,
$$

\footnotetext{
$\star$ Research supported in part by NSF under grant DMS-9400243 (DMS-9596041)
} 
which is now considered a classical fact, and all energy minimizers saturating the inequality (2) can be explicitly constructed in terms of rational functions, which thus renders the model integrable

Since the functional (1) is invariant under $O(3)$, we may always assume that the asymptotic state $\phi_{\infty}$ of a finite-energy field configuration $\phi$ is simply, say, $\mathbf{n}=(0,0,1)$ (the north pole) This choice breaks the $O(3)$ symmetry down to $S O(2)=U(1)$ which is clearly represented by the elements of $O(3)$ that preserve the direction $\mathbf{n}$. Consequently one is tempted to include a minimal potential term like $(1-\mathbf{n} \cdot \phi)^{2}$ in the energy integral to automatically respect such a choice of the asymptotics However, although the new energy is still clearly bounded from below and satisfies (2), a simple scaling argument indicates that there can be no nontrivial solutions To overcome this difficulty, an important device in gauge theory may be introduced so that an extra gauge vector field $\mathbf{A}=\left(A_{1}, A_{2}\right)$ is added, the usual partial derivatives in (1) are replaced by the corresponding gauge-covariant derivatives, $D_{1}, D_{2}$, and the energy contribution of $\mathbf{A}$, measured by the associated curvature $B=\partial_{1} A_{2}-\partial_{2} A_{1}$, is counted as a part of the total energy. Now the energy reads

$$
E(\phi, \mathbf{A})=\frac{1}{2} \int\left(D_{1} \phi\right)^{2}+\left(D_{2} \phi\right)^{2}+(1-\mathbf{n} \cdot \phi)^{2}+B^{2},
$$

where $D_{1} \phi=\partial_{j} \phi+A_{j}(\mathbf{n} \times \phi), j=1,2$.

The model (3) was first introduced in the recent work of Schroers [3] as an approximate soliton model for heavy particles and is connected to the baby Skyrme model (see [4] and the references therein) and Higgs vortices [5,6].

Following [3] we identify the curvature $B$ as the magnetic field and consider the magnetic flux

$$
\Phi=\Phi(\phi, \mathbf{A})=\int B
$$

In [3] Schroers compares the flux here with those in various vortex systems in which quantized flux implies topological stability (e g., the Abelian Higgs vortices $[5,7]$ ) while nonquantized flux implies otherwise (e.g., the nontopological ChernSimons vortices $[6,8,9])$. However, Schroers points out in [3] that the topological stability of the solitons given in the model (3) is independent of their magnetic flux. These solitons can carry arbitrary flux but yet be topologically stable. It is this last interesting property that motivates the rigorous study of the present paper We will show indeed that there are solutions at various quantized minimal energy levels, and at each energy level, there are solutions whose magnetic fluxes can assume arbitrary values from a designated open interval.

Recall that we can express $\operatorname{deg}(\phi)$ as

$$
\operatorname{deg}(\phi)=\frac{1}{4 \pi} \int \phi \cdot\left(\partial_{1} \phi \times \partial_{2} \phi\right)
$$

One of the crucial steps in [3] is to apply boundary behavior of a finite-energy field configuration to rewrite (5) into the useful form

$$
\operatorname{deg}(\phi)=\frac{1}{4 \pi} \int \phi \cdot\left(D_{1} \phi \times D_{2} \phi\right)+B(1-\mathbf{n} \cdot \phi) .
$$

Thus, in view of (6), the energy (3) can be recast into the form

$$
E(\phi, \mathbf{A})=4 \pi|\operatorname{deg}(\phi)|+\frac{1}{2} \int\left(D_{1} \phi \pm \phi \times D_{2} \phi\right)^{2}+(B \mp[1-\mathbf{n} \cdot \phi])^{2},
$$


according to the sign of $\operatorname{deg}(\phi)$. Consequently, as (2) for the $O(3)$ sigma model, Schroers [3] arrives at the conclusion that the energy (3) has the same Bogomol'nyi type topological lower bound and that this bound (2) is attained if and only if $(\phi, \mathbf{A})$ solves the self-dual Bogomol'nyi equations

$$
\begin{aligned}
D_{1} \phi & =-\phi \times D_{2} \phi, \\
B & =1-\mathbf{n} \cdot \phi, \\
\phi & : \mathbf{R}^{2} \rightarrow S^{2} .
\end{aligned}
$$

Here and in the sequel we concentrate on the (self-dual) case that $\operatorname{deg}(\phi)=$ $N \geqq 0$ because the anti-self-dual case $\operatorname{deg}(\phi)<0$ can be obtained by a simple transformation.

Note that, unlike the classical $O(3)$ model, the system (8) cannot be solved explicitly and there are some new features embedded. For example, Schroers [3] already found that there is no radially symmetric $N=1$ solution. Further understanding of (8) can only come from nonlinear functional analysis.

Since $\phi \in S^{2}$ we have $-1 \leqq \mathbf{n} \cdot \phi \leqq 1$. Inserting this property in the second equation in (8) we see that the magnetic field $B$ satisfies $0 \leqq B \leqq 2$. In other words the flux-lines are penetrating the plane along the same direction and the locations of the spots where the magnetic field achieves the greatest possible penetration is given by those points $p \in \mathbf{R}^{2}$ at which

$$
B(p)=B_{\max }=2 \text {. }
$$

Of course, due to the structure of the second equation in (8), the locations of the peaks of the magnetic field given in (9) are equivalently represented by the valleys of the projection of the map $\phi$ along $\mathbf{n}$, at which

$$
\mathbf{n} \cdot \phi(p)=(\mathbf{n} \cdot \phi)_{\min }=-1 \text {. }
$$

Such a simple identification leads to the important observation that the number of points at which the peaks or valleys take place is actually the degree $N$ of the map $\phi$. To see this fact we use $\mathbf{s}=(0,0,-1)$ to denote the south pole of $S^{2}$ and express the set of the locations of valleys defined in (10) as

$$
P=\phi^{-1}(\mathbf{s}) \text {. }
$$

It will be seen that $\# P$ (the number of points) $=N=\operatorname{deg}(\phi)$ which is a topological invariant. We now state and comment on our main results.

Theorem 1. The system (8) has no finite-energy degree one solutions whatsoever.

This result is the general form of Schroers' finding that there can be no radially symmetric degree one solutions since we have removed the radial symmetry restriction for the solutions. Such a nonexistence property alone indicates that the model here indeed possesses some new features.

The above theorem says that to obtain existence of solutions we need to consider the case that the degree of the map $\phi$ is greater than one. We have

Theorem 2. For any $N \geqq 2$ the system (8) has a family of finite-energy smooth solutions containing at least $(4 N-3)$ independent continuous parameters to fulfill the topological condition $\operatorname{deg}(\phi)=N$.

In fact this theorem may be stated in the following precise form. 
Theorem 3. For any integers $M, N$ satisfying $N \geqq 2, M<N-1$, the parameter $\alpha$ in the range

$$
1-\frac{M+2}{N}<\alpha<1-\frac{M+1}{N}
$$

and the points $p_{1}, p_{2}, \ldots, p_{N}, q_{1}, q_{2}, \ldots, q_{M} \in \mathbf{R}^{2}$ which may appear repeatedly in the collection, the system (8) has a solution $\left(\phi_{(\alpha)}, \mathbf{A}_{(x)}\right)$ so that

$$
\left\{p_{1}, p_{2}, \ldots, p_{N}\right\}=\phi_{(\gamma)}^{-1}(\mathbf{s}), \quad\left\{q_{1}, q_{2}, \ldots, q_{M}\right\}=\phi_{(\gamma)}^{-1}(\mathbf{n}),
$$

the energy lower bound (2) is saturated at the quantized level,

$$
E\left(\phi_{(\alpha)}, \mathbf{A}_{(x)}\right)=4 \pi N
$$

with $\operatorname{deg}\left(\phi_{(\alpha)}\right)=N$, and the total magnetic flux (4) is given by

$$
\Phi\left(\phi_{(\alpha)}, \mathbf{A}_{(\alpha)}\right)=2 \pi N \alpha .
$$

Furthermore, set

$$
\beta=2(N-M)-2 N \alpha
$$

Then the solution decays at infinity according to

$$
\begin{aligned}
\phi_{1}^{2}, \phi_{2}^{2}, 1-\phi_{3}^{2} & =\mathrm{O}\left(|x|^{-\beta}\right), \\
B=1-\mathbf{n} \cdot \phi & =\mathrm{O}\left(|x|^{-\beta}\right), \\
\left|D_{1} \phi\right|^{2}+\left|D_{2} \phi\right|^{2} & =\mathrm{o}\left(|x|^{-\beta}\right) .
\end{aligned}
$$

In other words, for given integer $N \geqq 2$ and the points $p_{1}, p_{2}, \ldots, p_{N} \in \mathbf{R}^{2}$, the system (8) has a solution so that these points p's are realized as the locations of the maximal peaks of the magnetic field and the total energy is proportional to the number $N$ which may well be viewed as the number of particles in the system Besides, the magnetic flux (14) can be designated in a continuous interval by using the parameter $\alpha$ in (12) and the decay exponent $\beta$ in (16) can also be specified in the continuous range $2<\beta<2(N-M)$ so that $\alpha$ and $\beta$ are related to each other through (15).

The count of the number of parameters stated in Theorem 2 now follows easily from Theorem 3. The points $p_{1}, p_{2}, ., p_{N}$ give $2 N$ parameters which are simply their coordinates, the points $q_{1}, q_{2}, \ldots, q_{M}$ can give up to $2(N-2)$ parameters because we may have $M=N-2$; the parameter $\alpha$ or $\beta$ gives one more degree of freedom

In the Ginzburg-Landau theory of superconductivity the greatest penetration of the magnetic field occurs at the zeros of the order parameter where the normal phase is restored. Such a phenomenon is known as the Meissner effect. In our gauged sigma model, however, the greatest penetration of the magnetic field $B$ occurs at the point set $\phi^{-1}(\mathbf{s})$ in $\mathbf{R}^{2}$ If $B$ is viewed as a vorticity field the system clearly represents multivortices If $B$ is viewed as a physical force such as the magnetic field the system represents multisolitons which may be thought of as a collection of identical particles because the maximal peaks of $B$ indicate well-defined force concentration at those points The surprising feature of the solution is that if the points $p_{1}, p_{2}, \ldots, p_{N}$ 
are viewed as magnetic monopoles, then the points $q_{1}, q_{2}, \ldots, q_{M}$ behave like antimonopoles. This phenomenon may be seen from (12) and (14): Although, according to the second equation in (8) the magnetic field at $p$ 's and $q$ 's have the same sign (in fact the magnetic field at the points $q$ 's vanishes), the formula (12) says that the presence of the points $q$ 's reduces the total flux. Besides, by (13), the total energy only depends on the number $N$ of the points $p$ 's but not the number $M$ of the points $q$ 's. When $M=0$ but $N$ is large, the expression (14) gives rise to a flux which is close to what is to be expected in the Ginzburg-Landau theory.

To conclude this section, we also notice that Theorem 1 is in fact a consequence of the following more general statement.

Theorem 4. Let $N$ be an integer. Then (8) has a finite-energy solution $(\phi, \mathbf{A})$ with $\operatorname{deg}(\phi)=N$ and $\# \phi^{-1}(\mathbf{n})=M$ if and only if $N-2 \geqq M$.

Since $M \geqq 0$ it is seen that there is a degree $N$ solution if and only if $N \geqq 2$ and Theorem 1 follows immediately.

In the rest of the paper we prove the above results. In the next section we follow [3] to rewrite (8) into an elliptic scalar equation with some Dirac function type source terms representing vortices and anti-vortices. In Sect. 3 we carry out the existence proof for part of Theorem 2 or 3 using a suitable weighted Sobolev space setting and find the restricted ranges of the free parameters. In Sect. 4 we establish the asymptotic behavior of the solution obtained by using comparison functions. In Sect. 5 we observe that the degree associated with the solution $(\phi, \mathbf{A})$ is actually the number $\# \phi^{-1}(\mathbf{s})$ and complete the proof of Theorem 2 or 3 . In Sect. 6 we prove Theorem 4 and complete the paper with some remarks.

\section{Multisolitons and the Reduced Governing Equations}

As in [3], consider the stereographic projection from the south pole $\mathbf{s}$ of $S^{2}$. We can then represent $\phi$, at least partially, as a map $\mathbf{u}=\left(u_{1}, u_{2}\right): \mathbf{R}^{2} \rightarrow \mathbf{R}^{2}$,

$$
u_{1}=\frac{\phi_{1}}{1+\phi_{3}}, \quad u_{2}=\frac{\phi_{2}}{1+\phi_{3}}
$$

where, of course,

$$
\phi_{3}= \begin{cases}\sqrt{1-\phi_{1}^{2}-\phi_{2}^{2}} & \text { when } \phi \text { lies on the upper sphere } S_{+}^{2}, \\ -\sqrt{1-\phi_{1}^{2}-\phi_{2}^{2}} & \text { when } \phi \text { lies on the lower sphere } S_{-}^{2} .\end{cases}
$$

Thus the transformation (17) is not well defined at the south pole $\phi_{1}=0, \phi_{2}=0$, $\phi_{3}=-1$. In fact, let

$$
p \in P=\phi^{-1}(\mathbf{s}) \text {. }
$$

Then by L'Hôpital's rule we easily see that the continuity of $\phi$ at $x=p$ implies the interesting property

$$
\lim _{x \rightarrow p}|\mathbf{u}(x)|^{2}=\lim _{x \rightarrow p}\left(u_{1}^{2}+u_{2}^{2}\right)=\lim _{x \rightarrow p} \frac{\phi_{1}^{2}+\phi_{2}^{2}}{\left(1-\sqrt{1-\left[\phi_{1}^{2}+\phi_{2}^{2}\right]}\right)^{2}}=\infty .
$$


In order to explore (20) further, we recall that, away from those points given in (19), the complex function $u=u_{1}+\mathrm{i} u_{2}$ reduces the Bogomol'nyi system into the following more familiar "vortex" equations [3]

$$
\begin{gathered}
D_{1} u=-\mathrm{i} D_{2} u, \\
B=\frac{2|u|^{2}}{1+|u|^{2}}, x \in \mathbf{R}^{2}, \quad x \notin P=\phi^{-1}(\mathbf{s}) \\
D_{j} u=\partial_{j} u+\mathrm{i} A_{j} u, \quad j=1,2,
\end{gathered}
$$

Thus, using the usual $\partial^{*}$-lemma argument as in [7], we see that near a point $p \in P$ the function $u$ may be written as the product of a nonvanishing function and a meromorphic function with $p$ as the only singular point In fact $p$ is a pole. To see this, we consider $U=1 / u$ near $p$ Then (20) says that $U$ is continuous at $p$ and $U(p)=0$ Furthermore, simple calculation gives us

$$
D_{j}^{*} U=-U^{2} D_{j} u, \quad j=1,2, x \neq p .
$$

Here $D_{j}^{*} U=\left(\partial_{j}-\mathrm{i} A_{j}\right) U$ Using the first equation in (21) we have $D_{1}^{*} U=-\mathrm{i} D_{2}^{*} U$ Therefore the above argument and the removable singularity theorem tell us that, near $p, U$ is the product of a nonvanishing function and a holomorphic function Hence $p$ must be a zero of $U$ with an integer multiplicity Such a property is crucial for the rest of the work here.

In general, assume

$$
P=\phi^{-1}(\mathbf{s})=\left\{p_{1}, p_{2}, \quad, p_{k}\right\} .
$$

Then there are positive integers $n_{1}, n_{2}, \quad, n_{k}$ so that, up to a positive factor,

$$
|u(x)|=\frac{1}{\left|x-p_{,}\right|^{n_{1}}}, \quad j=1,2, \ldots, k
$$

Besides we assume that $\phi^{-1}(\mathbf{n})$ is finite and set

$$
Q=\phi^{-1}(\mathbf{n})=\left\{q_{1}, q_{2}, \ldots, q_{l}\right\}
$$

Then $\phi_{1}\left(q_{j}\right)=0, \phi_{2}\left(q_{j}\right)=0, \phi_{3}\left(q_{j}\right)=1$, and, so, $u\left(q_{j}\right)=0, j=1,2, \ldots, l$ Consequently there are positive integers $m_{1}, m_{2}, \quad, m_{l}$ so that, up to a positive factor,

$$
|u(x)|=\left|x-q_{1}\right|^{m_{1}}, \quad j=1,2, ., l
$$

Finally, using the information (22)-(25) and the new variable $v=\ln |u|^{2}$ in (21), we see that the system (21) is reduced to the following scalar equation with the Dirac function type point source terms

$$
\Delta v=\frac{4 e^{v}}{1+e^{v}}-4 \pi \sum_{j=1}^{k} n_{j} \delta_{p_{j}}+4 \pi \sum_{j=1}^{l} m_{j} \delta_{q_{l}}, \quad x \in \mathbf{R}^{2}
$$

Equation (26) is a typical planar "vortex" equation. Its most interesting feature is that vortices of opposite charges (at $p_{j}$ 's and $q_{j}$ 's, respectively) co-exist Although the vorticity or magnetic field $B$ does not change its sign the vortices at $p_{j}$ 's may still be called anti-vortices, whereas, at $q_{j}$ 's, vortices

The next section is an analytic study of (26) Since finite-energy condition $\phi(x) \rightarrow \mathbf{n}$ as $|x| \rightarrow \infty$ requires $u(x) \rightarrow 0$ as $|x| \rightarrow \infty$, we need to look for solutions of (26) satisfying $v(x) \rightarrow-\infty$ as $|x| \rightarrow \infty$ 


\section{Proof of Existence}

Our main strategy is to construct suitable lower and upper solutions of (26). At the first glance, (26) allows a variational principle. However, the structure of the problem makes it hard to control some important terms. Thus we turn to apply similar variational principles to get lower and upper solutions as approximations. When this is done, an exact solution is ensured provided that the obtained upper solution is larger than the lower solution indeed.

First, introduce the background functions

$$
v_{1}=-\sum_{j=1}^{k} n_{j} \ln \left(1+\left|x-p_{j}\right|^{-2}\right), \quad v_{2}=-\sum_{j=1}^{l} m_{j} \ln \left(1+\left|x-q_{j}\right|^{-2}\right),
$$

and

$$
g_{1}=4 \sum_{j=1}^{k} n_{j}\left(1+\left|x-p_{j}\right|^{2}\right)^{-2}, \quad g_{2}=4 \sum_{j=1}^{l} m_{j}\left(1+\left|x-q_{j}\right|^{2}\right)^{-2} .
$$

Set $v=-v_{1}+v_{2}+V$ in (26) and $N=\sum n_{j}, M=\sum m_{j}$. Then $V$ satisfies

$$
\Delta V=\frac{4 e^{-v_{1}+v_{2}+V}}{1+e^{-v_{1}+v_{2}+V}}-g_{1}+g_{2}
$$

with

$$
\int g_{1}=4 \pi N, \quad \int g_{2}=4 \pi M .
$$

Recall that we need to obtain a solution $V$ of (28) satisfying $V(x) \rightarrow-\infty$ as $|x| \rightarrow \infty$. For this purpose, we choose a function $v_{3} \in C^{\infty}\left(\mathbf{R}^{2}\right)$ so that

$$
v_{3}(x)=-\ln |x|, \quad|x| \geqq 1, \quad x \in \mathbf{R}^{2} .
$$

Then an integration by parts gives

$$
-\int \Delta v_{3}=2 \pi
$$

Let $\beta>0$ and

$$
f=-v_{1}+v_{2}, \quad K=e^{\beta v_{3}} .
$$

Then $V=\beta v_{3}+w$ transforms (28) into the equation

$$
\Delta w=\frac{4 K e^{f+w}}{1+K e^{f+w}}-g,
$$

where

$$
g=g_{1}-g_{2}+\beta \Delta v_{3} .
$$

It is important to record the space average of $g$ here in view of (29) and (30):

$$
\int g=2 \pi(2[N-M]-\beta) .
$$

It is also important to observe that

$$
\begin{aligned}
& g(x)=\mathrm{O}\left(|x|^{-4}\right), \\
& K(x)=\mathrm{O}\left(|x|^{-\beta}\right),
\end{aligned}
$$


In our variational treatment, we shall use $K$ as a weight function where we need the condition $\beta>2$ (see (34)) Besides, for some technical reason, we also need (33) to be positive. These restrictions make it clear that we must choose $\beta$ to satisfy

$$
2<\beta<2(N-M) .
$$

We will always observe (35) from now on. ${ }^{1}$

We denote the usual $L^{p}$ space over $\mathbf{R}^{2}$ simply by $L^{p}$ and its norm by \|\|$_{p}$. Define the weighted measure $d \mu=K d x$ and use $L^{p}(d \mu)$ to denote the induced $L^{p}$ space Let $\mathscr{H}$ be the space of $L^{2}$ functions $w$ such that

$$
\|w\|_{\mathscr{H}}^{2}=\|\nabla w\|_{2}^{2}+\|w\|_{L^{2}(d \mu)}^{2}<\infty .
$$

Then $\mathscr{H}$ contains all constant functions and, thus,

$$
\mathscr{H}^{\prime}=\left\{w \in \mathscr{H} \mid \int w d \mu=0\right\}
$$

is a closed subspace of $\mathscr{H}$. Recall that there is a constant $\gamma>0$ depending on $\beta$ given in (34) so that the following Trudinger--Moser type inequality holds [10]:

$$
\int e^{|w|} d \mu \leqq C e^{i\|\nabla\|_{2}^{2}}, \quad w \in \mathscr{H}^{\prime}
$$

For our problem the range of $\gamma$ will not be important.

It is interesting to note that $\mathrm{Eq}$ (31) allows a variational formulation based on the weighted Sobolev space $\mathscr{H}$ However, due to the fact that $g$ defined in (32) can change sign, it is difficult to control the quantity

$$
\int w d \mu
$$

for a minimizing sequence. Fortunately we may use a less direct approach to solve (31) To see this we first modify (31) into the form

$$
\Delta w=\frac{4 K e^{f+w}}{1+K e^{f+w}}-|g| .
$$

Of course a solution of (37) can serve as an upper solution of (31) Later we will show that it is easy to get a lower solution of (31) that can be chosen to stay below any upper solution obtained from (37). Thus we are led to a resolution of (31) by standard iterative methods. Consequently we can concentrate on (37) first

To solve (37) consider the functional

$$
I(w)=\int\left\{\frac{1}{2}|\nabla w|^{2}+4 \ln \left(1+K e^{f+w}\right)-|g| w\right\}, \quad w \in \mathscr{H}
$$

We shall look for a solution of (37) satisfying the constraint $\int \Delta w=0$, which we will need in, e g., Lemma 13. Thus we introduce the admissible class

$$
\mathscr{A}=\left\{w \in \mathscr{H}\left|\int \frac{K e^{f+w}}{1+K e^{f+w}}=\frac{1}{4} \int\right| g \mid\right\}
$$

\footnotetext{
${ }^{1}$ This condition is actually also almost necessary See the Note Added at the end of the paper
} 
Lemma 5. If $w$ solves the optimization problem

$$
\min \{I(w) \mid w \in \mathscr{A}\}
$$

then $w$ is a solution of Eq. (37).

Proof. By the rule of the Lagrange multipliers we have

$$
\int \nabla w \cdot \nabla \xi+\frac{4 K e^{f+w}}{1+K e^{f+w}} \xi-|g| \xi=\lambda \int \frac{K e^{f+w}}{\left(1+K e^{f+w}\right)^{2}} \xi,
$$

where $\lambda \in \mathbf{R}$ is a constant and $\xi \in \mathscr{H}$ is an arbitrary trial function. Let $\xi \equiv 1$ in (40). Using the definition of $\mathscr{A}$ and $w \in \mathscr{A}$ we immediately obtain $\lambda=0$. Returning to (40) with $\lambda=0$ we see that $w$ is a weak solution of (37). By the standard elliptic theory we find that $w$ is a classical solution of (37).

Lemma 6. The functional (38) is bounded from below in $\mathscr{A}$.

Proof. From the simple relation

$$
\ln \left(1+K e^{f+w}\right)=\left(f+\beta v_{3}+w\right)+\ln \left(1+e^{-\left(f+\beta v_{3}+w\right)}\right),
$$

we can rewrite (38) as

$$
\begin{aligned}
I(w)= & \frac{1}{2}\|\nabla w\|_{2}^{2}+4 \int \ln \left(1+K e^{f+w}\right)-\int|g| \ln \left(1+K e^{f+w}\right) \\
& +\int|g|\left(f+\beta v_{3}\right)+\int|g| \ln \left(1+e^{-\left(f+\beta v_{3}+w\right)}\right) .
\end{aligned}
$$

Of course the last two terms on the right-hand side of (41) are of no harm. The crucial terms are the first three terms.

Set

$$
W=\ln \left(1+K e^{f+w}\right) .
$$

Then $W \geqq 0$. Furthermore the definition of the functions $f, K$, the fact $w \in \mathscr{A}$, and the relation

$$
\nabla W=\frac{K e^{f+w}}{1+K e^{f+w}}\left(\nabla f+\beta \nabla v_{3}+\nabla w\right)
$$

imply the inequality

$$
\|\nabla W\|_{2} \leqq C_{1}\|\nabla w\|_{2}+C_{2},
$$

where, and in the sequel, we use $C_{1}, C_{2}$, etc. to denote various positive constants. Hence (41) leads to

$$
I(w) \geqq \frac{1}{4}\|\nabla w\|_{2}^{2}+C_{1}\|\nabla W\|_{2}^{2}+4 \int W-\int|g| W-C_{2} .
$$

On the other hand the property $W \geqq 0$ yields $W \geqq W^{2} /(1+W)$. So (42) becomes

$$
I(w) \geqq \frac{1}{4}\|\nabla w\|_{2}^{2}+C_{1}\|\nabla W\|_{2}^{2}+4 \int \frac{W^{2}}{1+W}-\int|g| W-C_{2} .
$$


We recall the standard interpolation inequality over $\mathbf{R}^{2}$ :

$$
\int W^{4} \leqq 2 \int W^{2} \int|\nabla W|^{2}
$$

Since $g \in L^{p}$ for any $p \geqq 1$ we can use (44) to obtain the bound

$$
\begin{aligned}
\int|g| W & \leqq\|g\|_{\frac{4}{3}}\|W\|_{4} \leqq \varepsilon\|W\|_{2}+C(\varepsilon)\|\nabla W\|_{2}+C \\
& \leqq \varepsilon\|W\|_{2}+\frac{C_{1}}{2}\|\nabla W\|_{2}^{2}+C(\varepsilon),
\end{aligned}
$$

where $C_{1}>0$ is as given in (43) and $\varepsilon>0$ is small.

Now, using (44) again,

$$
\begin{aligned}
\|W\|_{2}^{4} & =\left(\int \frac{W}{1+W}(1+W) W\right)^{2} \leqq \int \frac{W^{2}}{1+W} \int\left(W+W^{2}\right)^{2} \\
& \leqq 2 \int \frac{W^{2}}{1+W}\left(\int W^{2}+2 \int W^{2} \int|\nabla W|^{2}\right) .
\end{aligned}
$$

Consequently

$$
\|W\|_{2} \leqq 1+\int \frac{W^{2}}{1+W}+\|\nabla W\|_{2}^{2} .
$$

Inserting (45) and (46) into (43), we obtain the following important lower bound estimate

$$
I(w) \geqq \frac{1}{4}\|\nabla w\|_{2}^{2}+C_{1}\left(\|\nabla W\|_{2}^{2}+\int \frac{W^{2}}{1+W}\right)-C_{2} .
$$

Thus the lemma is proven

Lemma 7. Suppose that the parameter $\beta$ defined in $K$ or (34) satisfies $\beta \leqq 4$ Then the optimization problem (39) has a solution

Proof Let $w_{j} \in \mathscr{A}, j=1,2, \ldots$, be a minimizing sequence of (39) so that

$$
\lim _{J \rightarrow \infty} I\left(w_{J}\right)=\eta \equiv \min \{I(w) \mid w \in \mathscr{A}\} .
$$

Set $W_{j}=\ln \left(1+K e^{f+w_{j}}\right)$ Then (46) and (47) imply that $\left\{W_{l}\right\}$ is a bounded sequence in $L^{2}$.

On the other hand (42) implies that

$$
I\left(w_{1}\right) \geqq 4 \int W_{1}-\|g\|_{2}\left\|W_{j}\right\|_{2}-C
$$

Hence, using the $L^{2}$-boundedness of $\left\{W_{l}\right\}$ in (48), we conclude that the sequence $\left\{W_{j}\right\}$ is also bounded in $L^{1}\left(\mathbf{R}^{2}\right)$. It is useful to recall that (47) already implies that $\left\{\left\|\nabla w_{1}\right\|_{2}\right\}$ is bounded.

At this moment we need to invoke the following Poincaré type inequality on $\mathscr{H}$.

$$
\int w^{2} d \mu \leqq C \int|\nabla w|^{2}, \quad w \in \mathscr{H}^{\prime}
$$

For a proof see [10] 
We decompose $w_{j}$ into the form

$$
w_{j}=\bar{w}_{j}+w_{j}^{\prime}, \quad \bar{w}_{j} \in \mathbf{R}, \quad w_{j}^{\prime} \in \mathscr{H}^{\prime} .
$$

Inserting (50) into $I\left(w_{j}\right)$, we have

$$
\frac{1}{2}\left\|\nabla w_{j}\right\|_{2}^{2}+4\left\|W_{j}\right\|_{1}-\bar{w}_{j} \int|g|-\int|g| w_{j}^{\prime} \geqq \eta .
$$

Namely,

$$
\bar{w}_{j}\|g\|_{1} \leqq \frac{1}{2}\left\|\nabla w_{j}\right\|_{2}^{2}+4\left\|W_{j}\right\|_{1}+C\left\|g e^{-\left(f+\beta v_{3}\right)}\right\|_{\infty}\left\|w_{j}^{\prime}\right\|_{L^{2}(d \mu)}+|\eta| .
$$

Applying the Poincaré inequality (49) and the conditions (34) and $\beta \leqq 4$ in (51) we conclude immediately that $\left\{\bar{w}_{j}\right\}$ is bounded from above.

Moreover, since $w_{j} \in \mathscr{A}$, we have

$$
e^{\bar{w}_{J}} \int e^{f+w_{J}^{\prime}} d \mu>\int \frac{K e^{f+w_{j}}}{1+K e^{f+w_{j}}}=\int|g|
$$

Hence

$$
\bar{w}_{j}>\ln \left(\int|g|\right)-\ln \left(\int e^{f+w_{\jmath}^{\prime}} d \mu\right) \geqq \ln \left(\int|g|\right)-C_{1}\left\|\nabla w_{j}^{\prime}\right\|_{2}^{2}-C_{2}
$$

by (36). But we have shown that $\left\{\left\|\nabla w_{j}^{\prime}\right\|\right\}$ is bounded. Thus $\left\{\bar{w}_{j}\right\}$ is also bounded from below.

In summary we have shown that for the minimizing sequence $\left\{w_{j}\right\}$ of (39) the sequence $\left\{\bar{w}_{j}\right\}$ is bounded.

From (47) and (49) we see that $\left\{w_{j}^{\prime}\right\}$ is bounded in $\mathscr{H}$. By going to a suitable subsequence if necessary, we may assume that there is an element $w \in \mathscr{H}$ so that

$$
w_{j}=\bar{w}_{j}+w_{j}^{\prime} \rightarrow w \quad \text { as } j \rightarrow \infty \text { weakly in } \mathscr{H} .
$$

Recall that the embedding $\mathscr{H} \rightarrow L^{2}(d \mu)$ is completely continuous (see [10]). So we may assume that $w_{j} \rightarrow w$ strongly in $L^{2}(d \mu)$.

To see that the constraint in the admissible space $\mathscr{A}$ is preserved, we note that

$$
\begin{aligned}
& \int\left|\frac{K e^{f+w_{j}}}{1+K e^{f+w_{j}}}-\frac{K e^{f+w}}{1+K e^{f+w}}\right| \leqq \int K e^{f+\left|w_{j}\right|+|w|}\left|w_{j}-w\right| \\
& \quad \leqq C\left\|w_{j}-w\right\|_{L^{2}(d \mu)} \sup _{j}\left(e^{\left|\bar{w}_{j}\right|+|\bar{w}|+C_{1}\left(\left\|\nabla w_{j}\right\|_{2}^{2}+\|\nabla w\|_{2}^{2}\right)}\right) .
\end{aligned}
$$

Besides we also have

$$
\int\left|\ln \left(1+K e^{f+w_{\jmath}}\right)-\ln \left(1+K e^{f+w}\right)\right| \leqq \int K e^{f+\left|w_{\jmath}\right|+|w|}\left|w_{j}-w\right| .
$$

Thus, using (52), we see that the functional $I$ defined in (38) is weakly lower semicontinuous on $\mathscr{H}$.

Consequently we obtain $I(w) \leqq \liminf I\left(w_{j}\right)=\eta$ and $w \in \mathscr{A}$. In other words $w$ is a solution of (39). Of course it also solves (37) by Lemma 5 .

We now turn to the construction of a lower solution of (31). 
Lemma 8. Under the condition (35) the equation

$$
\Delta w=4 K e^{f+w}-g
$$

has a smooth solution in $\mathscr{H}$ Moreover $w \rightarrow$ a constant at infinity

Proof. See [11] This is where we use the property $\int g>0$ according to (35).

Lemma 9. Let $w$ be a solution of (53) and $c<0$ an arbitrary constant Then $w_{c}=w+c$ is a lower solution of (31)

Proof. This is a straightforward fact because

$$
\Delta w_{c}=\Delta(w+c)=4 K e^{f+w}-g \geqq \frac{4 K e^{f+w_{c}}}{1+K e^{f+w_{c}}}-g .
$$

In order to establish suitable comparison between the upper and lower solutions just obtained we need to recall the following facts from the well-developed theory of weighted Sobolev spaces [10].

For $\delta \in \mathbf{R}$ and $s \in \mathbf{N}$ (the set of nonnegative integers) define $W_{s, \delta}^{2}$ to be the closure of the set of $C^{\infty}$ functions over $\mathbf{R}^{2}$ with compact supports under the norm

$$
\|\xi\|_{W_{s, \delta}^{2}}^{2}=\sum_{|\gamma| \leqq s}\left\|(1+|x|)^{\delta+\left|\gamma^{\prime}\right|} D^{\prime} \xi\right\|_{2}^{2}
$$

Let $C_{0}\left(\mathbf{R}^{2}\right)$ be the set of continuous functions on $\mathbf{R}^{2}$ vanishing at infinity.

Lemma 10. If $s>1$ and $\delta>-1$, then $W_{s, \delta}^{2} \subset C_{0}\left(\mathbf{R}^{2}\right)$

Lemma 11. For $-1<\delta<0$, the Laplace operator $\Delta: W_{2, \delta}^{2} \rightarrow W_{0, \delta+2}^{2}$ is $1-1$ and the range of $\triangle$ has the characterization

$$
\Delta\left(W_{2, \delta}^{2}\right)=\left\{F \in W_{0, \delta+2}^{2} \mid \int F=0\right\} .
$$

Lemma 12. If $\xi \in \mathscr{H}$ and $\Delta \xi=0$ then $\xi=$ constant

Lemma 13. If $\beta$ in Lemma 7 satisfies $\beta<4$ then the solution $w$ of (37) obtained in Lemmas 5 and 7 goes to a constant at infinity

Proof. Since $w$ solves (37) we write the right-hand side of (37) as $F$. Then $F \in$ $L\left(\mathbf{R}^{2}\right)$ and $\int F=0$. Besides it is straightforward to show that $F \in W_{0, \delta+2}^{2}$ with $\delta$ satisfying

$$
-1<\delta=-1+\frac{1}{2}(\beta-2)<0
$$

by virtue of the condition $\beta<4$. In fact we first have

$$
\int\left(1+|x|^{\delta+2}\right)^{2}\left(\frac{K e^{f+w}}{1+K e^{f+w}}\right)^{2} \leqq C \int e^{2 w} d \mu
$$

with

$$
C=\sup _{r \in \mathbf{R}^{2}}\left\{K(x)\left(1+|x|^{\delta+2}\right)^{2}\right\}
$$


The quantity $C$ is finite if $2(\delta+2)-\beta \leqq 0$. The least stringent value of $\beta$ is ensured by setting $\beta=2(\delta+2)$ or $\delta=-2+\beta / 2=-1+(\beta-2) / 2$ as given in (54). Since $-1<\delta<0$ it is already clear that $|g| \in W_{0, \delta+2}^{2}$. Therefore by Lemma 11 there is a $\tilde{\xi} \in W_{2, \delta}^{2}$ such that $\Delta \tilde{\xi}=F$. From Lemma $10 \tilde{\xi}$ vanishes at infinity. In particular $\tilde{\xi} \in$ $L^{2}(d \mu)$. By the fact that $\nabla \tilde{\xi} \in W_{0, \delta+1}^{2}$ and $\delta>-1$, we get $\nabla \tilde{\xi} \in L^{2}$. Consequently $\tilde{\xi} \in \mathscr{H}$. By $w \in \mathscr{A}$ we have $\Delta(\tilde{\xi}-w)=0$. Since $\tilde{\xi}-w \in \mathscr{H}$, we find $\tilde{\xi}-w=$ constant which proves that $w$ goes to a constant at infinity as expected. The lemma is proven.

We can now turn to the proof of existence for the original equation (31).

Let $w^{+}$be an upper solution of (31) obtained in Lemmas 5 and 7 as a solution of (37) and $w^{-}=w_{c}$ a lower solution obtained in Lemma 9. Suppose that $\beta<4$ as stated in Lemma 13. Then $w^{+}$and $w^{-}$approach respectively their finite limiting values at infinity. In particular they are bounded functions. Therefore we can choose the parameter $c<0$ in the definition of $w_{c}=w^{-}$(Lemma 9) to make $w^{-}<w^{+}$on the entire $\mathbf{R}^{2}$. Thus we can use the method of iterations (cf. [13]) to get a solution of (31) so that

$$
w^{-}<w<w^{+} \text {in } \mathbf{R}^{2}
$$

which proves the existence of a solution of (31).

To calculate the magnetic flux generated by the solution, we need to study the behavior of the solution at infinity. In particular we want to find the value of $\int \Delta w$. This part of work is carried out in the next section.

\section{Asymptotic Behavior of the Solution}

In order to calculate the exact value of the magnetic flux we must evaluate the integral

$$
\int \frac{4 K e^{f+w}}{1+K e^{f+w}}
$$

where $w$ is the solution of (31). Since $w$ is obtained from monotone iterations the value of (56) is not known in the process of construction and some analysis has to be carried out. The main goal of this section is to show that (56) is equal to $\int g$ which is explicitly known by virtue of (33).

We first show that the solution $w$ obtained above lies in $\mathscr{H}$. For this purpose let the cut-off function $\xi \in C^{\infty}\left(\mathbf{R}^{2}\right)$ be such that

$$
\xi=1 \quad \text { in }\{x|| x \mid \leqq 1\}, \quad \xi=0 \quad \text { in }\{x|| x \mid \geqq 2\}, \quad 0 \leqq \xi \leqq 1 \quad \text { everywhere. }
$$

Set $\xi_{\rho}(x)=\xi(x / \rho)(\rho>0)$. Multiplying (31) by $\xi_{\rho}^{2} w$ and integrating by parts we have

$$
\int \xi_{\rho}^{2}|\nabla w|^{2}=-2 \int \xi_{\rho} w\left(\nabla \xi_{\rho} \cdot \nabla w\right)-\int \xi_{\rho}^{2} w\left(\frac{4 K e^{f+w}}{1+K e^{f+w}}-g\right) .
$$

However the definition of $\xi_{\rho}$ gives us

$$
\int\left|\nabla \xi_{\rho}\right|^{2} \leqq \frac{1}{\rho^{2}} \sup _{\mathbf{R}^{2}}|\nabla \xi|^{2} \int_{|x| \leqq 2 \rho}=4 \pi \sup _{\mathbf{R}^{2}}|\nabla \xi|^{2} .
$$


(Another way of getting the above bound is to use the scaling invariance to arrive at $\int\left|\nabla \xi_{\rho}\right|^{2}=\int|\nabla \xi|^{2} \leqq 4 \pi \sup |\nabla \xi|^{2}$.) Inserting this result into (57) we find that

$$
\int \xi_{\rho}^{2}|\nabla w|^{2} \leqq C_{1}+C_{2} \int \xi_{\rho}^{2}\left|\frac{4 K e^{f+w}}{1+K e^{f+w}}-g\right|,
$$

where we have used the fact that $w$ is a bounded function (see (55)) and that $K$ enjoys the decay rate given in (34). Letting $\rho \rightarrow \infty$ in (58) we have $|\nabla w| \in L^{2}$. Consequently we obtain the relation $w \in \mathscr{H}$.

After the above preparation we turn to the following useful result.

Lemma 14. Suppose that $\beta<4$ and that $w$ is the solution of (31) obtained in the last section Then $\int \Delta w=0$ Furthermore $w$ goes to a constant and $\partial_{j} w \rightarrow 0$ $(j=1,2)$ at infinity and such $a w$ is unique

Proof From (31) we have

$$
\begin{aligned}
\Delta\left(\partial_{j} w\right) & =\frac{4 K e^{f+w}}{\left(1+K e^{f+w}\right)^{2}}\left(\partial_{j} f+\beta \partial_{j} v_{3}+\partial_{j} w\right)-\left(\hat{o}_{j} g\right) \\
& =\frac{4 K e^{f+w}}{\left(1+K e^{f+w}\right)^{2}}\left(\partial_{j} w\right)+\mathrm{O}\left(|x|^{-(1+\beta)}\right)
\end{aligned}
$$

Since we have shown that $\partial_{j} w \in L^{2}$ the $L^{2}$-estimates applied in (59) gives us $\partial_{j} w \in W^{2,2}$ In particular $\partial_{j} w \rightarrow 0$ as $|x| \rightarrow \infty$ as expected. Besides the well-known Sobolev embedding theorem tells us that $\partial_{j} w \in W^{1, t}$ for any $t>1$ since we are in two dimensions.

On the other hand we can use the cut-off function $\xi_{\rho}$ defined earlier to get the integral bound

$$
\begin{aligned}
& \left|\int \xi_{\rho} \Delta w\right| \leqq \int\left|\nabla \xi_{\rho} \cdot \nabla w\right| \leqq\left\|\nabla \xi_{\rho}\right\|_{s}\|\nabla w\|_{t} \\
& \leqq C \rho^{-(s-2) / s}\|\nabla w\|_{t},
\end{aligned}
$$

where $s, t>1$ are conjugate exponents Of course, in (60), we can choose $s>2$ Then, letting $\rho \rightarrow \infty$ in (60), we arrive at the desired result $\int \Delta w=0$.

Therefore integrating (31) and using (33) we immediately find that

$$
\int \frac{K e^{f+w}}{1+K e^{f+w}}=\frac{\pi}{2}(2[N-M]-\beta) .
$$

Next we denote the right-hand side of (31) by $F$. Then $\int F=0$ and we can apply the argument in the proof of Lemma 13 to show that $w$ indeed approaches some constant at infinity.

Finally let $w_{1}, w_{2}$ be two solutions of (31) which go to their respective limiting values at infinity Then using the maximum principle in the equation

$$
\Delta\left(w_{1}-w_{2}\right)=\frac{K e^{f+\xi}}{\left(1+K e^{f+\xi}\right)^{2}}\left(w_{1}-w_{2}\right),
$$

where $\xi$ lies between $w_{1}$ and $w_{2}$, we conclude that $w_{1} \equiv w_{2}$ and the lemma follows 
When we recover a solution $(\phi, \mathbf{A})$ of $(8)$ from $w$ we can get the decay estimates stated in Theorem 3. (The procedure of recovering a solution of (8) follows the standard path that first one assigns the phase angle of $u$, hence defines the complex field $u$ itself, and, the gauge vector field $\mathbf{A}$ in the solution pair $(u, \mathbf{A})$ of (21) according to the prescription

$$
\begin{gathered}
\theta(z)=-\sum_{j=1}^{k} \arg \left(z-p_{j}\right)+\sum_{j=1}^{l} \arg \left(z-q_{j}\right), \\
u(z)=\exp \left(\frac{1}{2} v(z)+\mathrm{i} \theta(z)\right), \\
A_{1}(z)=\Re\left\{2 \mathrm{i} \partial^{*} \ln u(z)\right\}, \quad A_{2}(z)=\Im\left\{2 \mathrm{i} \partial^{*} \ln u(z)\right\}, \\
z=x_{1}+\mathrm{i} x_{2}, \quad \partial=\frac{1}{2}\left(\partial_{1}-\mathrm{i} \partial_{2}\right),
\end{gathered}
$$

and, then, one obtains the solution pair $(\phi, \mathbf{A})$ by converting the formula (17).) In fact recall that

$$
|u|^{2}=e^{v}=K e^{f+w}=\mathrm{O}\left(|x|^{-\beta}\right) \text { for }|x| \text { large }
$$

is sharp because $w$ tends to a finite limiting value at infinity. Thus the first two results in (16) are verified. The last one involves some tedious algebraic calculations. Here we will be sketchy.

First we have the relation

$$
\left|D_{j} \phi\right|^{2}=\frac{4}{\left(|u|^{2}+1\right)^{2}}\left|D_{j} u\right|^{2}, \quad j=1,2
$$

where the operators $D_{j}$ 's mean different things on the two sides.

Besides it can be verified that

$$
\left|D_{j} u\right|^{2}=\frac{1}{4} e^{v}|\nabla v|^{2}
$$

Using (62)-(64) we see that the third result in (16) follows because $|\nabla v| \rightarrow 0$ as $|x| \rightarrow \infty$. We choose not to elaborate on the decay rate of $|\nabla w|$ (Lemma 14). Thus the asymptotic estimate for $\left|D_{j} \phi\right|^{2}(j=1,2)$ stated in (16) is rather crude.

Equation (61) easily leads to a calculation of the magnetic flux. In fact recall that $|u|^{2}=K e^{f+w}$. Therefore the second equation in (21) and (61) yield that

$$
\int B=2 \pi N \alpha, \quad \alpha=1-\frac{1}{N}\left(M+\frac{\beta}{2}\right) .
$$

Hence (14) and (15) are derived. Furthermore inserting (35) into (15) we obtain the range (12) for the flux parameter $\alpha$.

We leave the verification that $\operatorname{deg}(\phi)=N$ to the next section. 


\section{Calculation of the Degree of the Map}

Let $(u, \mathbf{A})$ be a solution of (21) constructed in the previous sections so that there are points $p_{1}, \ldots, p_{k} \in \mathbf{R}^{2}$ and $n_{1}, \ldots, n_{k} \in \mathbf{N}$ to make (23) hold. Put

$$
N=\sum n_{j}=n_{1}+\quad+n_{k} \text {. }
$$

With $u=u_{1}+\mathrm{i} u_{2}$ we can invert the transformation (17)-(18) to obtain a map $\phi=\left(\phi_{1}, \phi_{2}, \phi_{3}\right): \mathbf{R}^{2} \rightarrow S^{2}$ so that $\phi^{-1}(\mathbf{s})=\left\{p_{1}, \ldots, p_{k}\right\}=P$ (see (22)). Then, counting algebraic multiplicities, we have $\# \phi^{-1}(\mathbf{s})=N$ In the following we will show that $N=\operatorname{deg}(\phi)$ when $\phi$ is viewed as a map from $S^{2}$ to $S^{2}$ form

In fact, near the set $P$, we have the inversion of (17)-(18) from $u$ to $\phi$ in the

$$
\phi_{1}=\frac{2 u_{1}}{u_{1}^{2}+u_{2}^{2}+1}, \quad \phi_{2}=\frac{2 u_{2}}{u_{1}^{2}+u_{2}^{2}+1}
$$

For convenience we use the reciprocal of $u$,

$$
U=\frac{1}{u}=\frac{u_{1}}{u_{1}^{2}+u_{2}^{2}}-\mathrm{i} \frac{u_{2}}{u_{1}^{2}+u_{2}^{2}} \equiv U_{1}+\mathrm{i} U_{2},
$$

to study $\phi_{1}, \phi_{2}$ near the preimages (the set $P$ ) of the south pole under $\phi$. It is easily seen from (65) that

$$
\phi_{1}=\frac{2 U_{1}}{U_{1}^{2}+U_{2}^{2}+1}, \quad \phi_{2}=-\frac{2 U_{2}}{U_{1}^{2}+U_{2}^{2}+1} .
$$

It is clear that, at a pole $p \in P$ of $u$, we have $U(p)=0$ and $\phi_{1}(p)=\phi_{2}(p)=0$. Thus by virtue of (66) we have the expressions

$$
\frac{\partial \phi_{1}}{\partial x^{\prime}}(p)=2 \frac{\partial U_{1}}{\partial x^{\prime}}(p), \quad \frac{\partial \phi_{2}}{\partial x^{j}}(p)=-2 \frac{\partial U_{2}}{\partial x^{j}}(p), \quad j=1,2 .
$$

Since $U$ satisfies the equation $D_{1}^{*} U=-\mathrm{i} D_{2}^{*} U$ therefore we obtain the Jacobian of (66) at $p$ in the form

$$
\left.\frac{\partial\left(\phi_{1}, \phi_{2}\right)}{\partial\left(x^{1}, x^{2}\right)}\right|_{x=p}=-2\left(\left|\nabla U_{1}\right|^{2}+\left|\nabla U_{2}\right|^{2}\right)(p),
$$

which is nonvanishing if $p$ is a simple zero of $U$ or a simple pole of $u$ In this situation $p$ is a regular point of $\phi$.

For simplicity we assume first that the points in $P$ are all simple zeros of $U$ or simple poles of $u$ Then $P=\left\{p_{1}, p_{2}, . ., p_{N}\right\}$ and $n_{1}=n_{2}=\cdot \cdot=n_{N}=1$ Thus $P=\phi^{-1}(\mathbf{s})$ implies that the south pole $\mathbf{s}$ is a regular value of $\phi$. Furthermore, at any $p \in P$, we have

$$
\partial_{1} \phi \times \partial_{2} \phi=\left(0,0,\left.\frac{\partial\left(\phi_{1}, \phi_{2}\right)}{\partial\left(x^{1}, x^{2}\right)}\right|_{x=p}\right) .
$$

Hence by (67) we see that $\phi$ also preserves orientation at each $p$. This observation enables us to conclude that

$$
\operatorname{deg}(\phi)=\# \phi^{-1}(\mathbf{s})=N
$$


Next we consider the general case that the poles can have multiplicities. For this purpose we still use $p_{1}, p_{2}, \ldots, p_{N}$ to denote these points but we also understand that some of the points may coincide to form multiple poles. Then the solutions obtained are labelled by the multidimensional parameter vector $\mathbf{p}=\left(p_{1}, p_{2}, \ldots, p_{N}\right) \in \mathbf{R}^{2 N}$. We will show that the solutions continuously depend on $\mathbf{p}$. As a consequence of the homotopy invariance of the Hopf degree, the result (68) will follow naturally in general because the case of multiple poles may be approximated continuously by solutions of simple poles.

Lemma 15. Let $w$ be the solution of (31) obtained earlier and $\mathbf{p} \in \mathbf{R}^{2 N}$ the parameter vector indicating the locations of the poles. Then $w$ depends on $\mathbf{p}$ continuously in the $C(\Omega)$-norm for any bounded subdomain $\Omega$ of $\mathbf{R}^{2}$.

Proof. We denote the dependence of $w$ on $\mathbf{p}$ explicitly by $w_{\mathbf{p}}$. The uniqueness established in Lemma 14 means that such a notation has no ambiguity. Consider the set $B_{r}=\left\{\mathbf{p} \in \mathbf{R}^{2 N}|| \mathbf{p} \mid<r\right\}$ with $r>0$ arbitrary. We show that $w_{\mathbf{p}}$ depends on $\mathbf{p}$ continuously for $\mathbf{p} \in B_{r}$.

In fact we can find p-independent Lipschitz continuous functions $h_{1}, h_{2} \geqq 0$ on $\mathbf{R}^{2}$ so that

$$
h_{1} \leqq e^{f} \leqq h_{2} \quad \text { in } \mathbf{R}^{2}
$$

for any $\mathbf{p} \in B_{r}$. For example we may define

$$
\begin{array}{ll}
h_{1}(x)=\inf _{\mathbf{p} \in B_{r}}\left\{e^{f(x)}\right\} & \text { for }|x| \leqq r+1 \text { or }|x| \geqq r+2, \\
h_{2}(x)=\sup _{\mathbf{p} \in B_{r}}\left\{e^{f(x)}\right\} & \text { for }|x| \leqq r+1 \text { or }|x| \geqq r+2,
\end{array}
$$

and in the region $r+1<|x|<r+2$ we make suitable interpolations. Then we see as before that there are solutions $U$ and $V$ to the equations

$$
\Delta U=\frac{4 K h_{1} e^{U}}{1+K h_{1} e^{U}}-|g|
$$

and

$$
\Delta V=4 K h_{2} e^{V}-g
$$

respectively which approach their finite limiting values at infinity. Note that Eqs. (70) and (71) are p-independent. By virtue of (69) we have in addition that

$$
\Delta U \leqq \frac{4 K e^{f+U}}{1+K e^{f+U}}-g
$$

and

$$
\Delta(V+c) \geqq \frac{4 K e^{f+V+c}}{1+K e^{f+V+c}}-g
$$

for any $c<0$. Hence by choosing $c<0$ in (73) suitable we obtain from (72)-(73) a pair of upper and lower solutions of (31) for any $\mathbf{p} \in B_{r}, U$ and $V_{c}=V+c$, which we still denote by $V$, so that $U>V$ everywhere. Thus the uniqueness established in Lemma 14 implies that there hold the uniform bounds:

$$
V \leqq w_{\mathbf{p}} \leqq U \quad \text { in } \mathbf{R}^{2}
$$




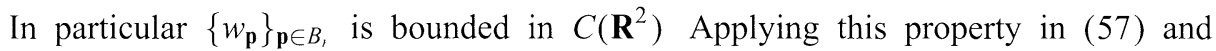
using the argument there we see that $\left\{\left|\nabla w_{\mathbf{p}}\right|\right\}$ is also bounded in $L^{2}$. By this result and (59) we conclude that $\left\{\partial_{j} w_{\mathbf{p}}\right\}(j=1,2)$ are bounded sets in $W^{2,2}$

Suppose that there is an $\Omega_{0} \subset \mathbf{R}^{2}$ and a point $\mathbf{p}_{0} \in B_{r}$ such that a sequence $\left\{\mathbf{p}_{k}\right\}$ may be found to make

$$
\left\|w_{\mathbf{p}_{h}}-w_{\mathbf{p}_{0}}\right\|_{C\left(\Omega_{0}\right)} \geqq \varepsilon_{0}, \quad k=1,2, \ldots
$$

for some number $\varepsilon_{0}>0$ but $\mathbf{p}_{k} \rightarrow \mathbf{p}_{0}$ as $k \rightarrow \infty$.

However since the boundedness properties of $\left\{w_{\mathbf{p}}\right\}$ and their derivatives under the appropriate norms found above enables us to conclude that, without loss of generality, we may assume that $w_{\mathbf{p}_{h}} \rightarrow w$ as $k \rightarrow \infty$ weakly in $\mathscr{H}$ so that $w$ is a solution of (31). Using Lemma 14 we have $w=w_{\mathbf{p}_{0}}$.

On the other hand, since $\left\{w_{\mathbf{p}_{k}}\right\}$ is weakly convergent to $w$ in $W^{2,2}\left(\Omega_{0}\right)$, the compact embedding theorem says that the strong convergence holds in $C\left(\Omega_{0}\right)$. Such a result violates (76) because $w=w_{\mathbf{p}_{0}}$ already.

The lemma is proven.

Recall that, as a component of the solution to the system (21), the complex function $u_{\mathrm{p}}$ is related to the solution $w_{\mathbf{p}}$ of (31) by

$$
\left|u_{\mathbf{p}}\right|^{2}=K e^{f+w_{\mathbf{p}}} .
$$

Since $K=\mathrm{O}\left(|x|^{-\beta}\right)$ is independent of $\mathbf{p}$ and $e^{f+w_{\mathrm{p}}}$ is uniformly bounded for $\mathbf{p} \in B_{l}$ (see (74)) it is clear that $\left|u_{\mathbf{p}}\right|^{2}$ goes to zero uniformly fast as $|x| \rightarrow \infty$ for $\mathbf{p} \in B_{l}$. Returning to the original map $\phi_{\mathbf{p}}$ we see that $\phi_{\mathbf{p}}$ goes to the north pole $\mathbf{n}$ uniformly fast as $|x| \rightarrow \infty$ for all $\mathbf{p} \in B_{\text {, }}$. In fact there holds the uniform rate of convergence

$$
\left|\phi_{\mathbf{p}}(x)-\mathbf{n}\right| \leqq C|x|^{-\beta}, \quad \forall x \in \mathbf{R}^{2},
$$

where $C>0$ is an absolute constant This result indicates that the map $B_{r} \rightarrow C\left(S^{2}\right)$, $\mathbf{p} \mapsto \phi_{\mathbf{p}}$ is indeed continuous. Consequently (68) must hold in general where a preimage with multiplicity $k$ is counted $k$ times.

In summary we have identified the integer $N$, the number of the prescribed poles of the complex function $u$ in the solution of the Bogomol'nyi system (5), to be the topological degree of the map $\phi$ as expected

\section{Proof of Nonexistence}

The condition (35) says that we need $N \geqq M+2$ to ensure the existence of a solution of (26) that gives rise to a solution $(\phi, \mathbf{A})$ of the Bogomol'nyi system (8). Recall that the integer $N$ is actually the number of the preimages of the south pole $\mathbf{s}$ under the map $\phi$ and $M$ is the number of the preimages of the north pole $\mathbf{n}$ under $\phi$, both counting algebraic multiplicities In this section we show that this condition is also necessary In other words we prove that whenever $N<M+2$ (or $N \leqq M+1)$ there can be no solutions over $\mathbf{R}^{2}$ for Eq (26) We will see that such a result implies the important conclusion that the system (8) has no solution with $\operatorname{deg}(\phi)=1$

Suppose otherwise that the Bogomol'nyi equations (8) have a multisoliton so-

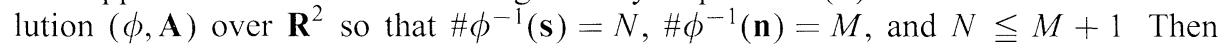


the stereographic projection (17) and the variable $v=\ln |u|^{2}$ leads us to (26) with $N=\sum n_{j} \geqq 0$ and $M=\sum m_{j} \geqq 0$. In (26) set now

$$
v(x)=-2 \sum_{j=1}^{k} n_{j} \ln \left|x-p_{j}\right|+2 \sum_{j=1}^{l} \ln \left|x-q_{j}\right|+w(x) .
$$

Then (26) becomes

$$
\Delta w=\frac{4 K_{0} e^{w}}{1+K_{0} e^{w}} \equiv h(x), \quad K_{0}(x)=\prod_{j=1}^{k}\left|x-p_{j}\right|^{-2 n_{j}} \prod_{j=1}^{l}\left|x-q_{j}\right|^{2 m_{J}} .
$$

Recall that

$$
K_{0} e^{w}=e^{v}=|u|^{2} .
$$

By virtue of (17)-(18) we have for any

$$
r_{1}>\max _{j}\left\{\left|p_{j}\right|\right\}
$$

a bound $C\left(r_{1}\right)>0$ for $|u|^{2}$ away from the points $p_{1}, p_{2}, \ldots, p_{k}$ in the form

$$
|u(x)|^{2} \leqq C\left(r_{1}\right), \quad x \in \mathbf{R}^{2}, \quad|x| \geqq r_{1} .
$$

For (76) we now proceed as in [14].

Consider the polar coordinates $(\rho, \theta)$ for $\mathbf{R}^{2}$. Integrating (76) in the region $0 \leqq$ $\rho \leqq r, 0 \leqq \theta \leqq 2 \pi$ we have

$$
\int_{0}^{r} \int_{0}^{2 \pi}(\Delta w) \rho d \theta d \rho=\int_{0}^{r} \int_{0}^{2 \pi} h \rho d \theta d \rho
$$

Set

$$
W(r)=\frac{1}{2 \pi} \int_{0}^{2 \pi} w(r, \theta) d \theta .
$$

With the notation (81) we are led from $(80)$ by the $2 \pi$-periodicity of $w$ the following equation:

$$
r W_{r}(r)=\frac{1}{2 \pi} \int_{0}^{r} \int_{0}^{2 \pi}\left(\left[\rho w_{\rho}\right]_{\rho}+\frac{1}{\rho} \frac{\partial^{2} w}{\partial^{2} \theta}\right) d \theta d \rho=\frac{1}{2 \pi} \int_{0}^{r} \int_{0}^{2 \pi} h \rho d \theta d \rho,
$$

where $W_{r}=d W / d r$. Differentiating (82) with respect to $r>0$ we find

$$
\frac{1}{r}\left(r W_{r}\right)_{r}=\frac{1}{2 \pi} \int_{0}^{2 \pi} h d \theta \text {. }
$$

On the other hand, choose $r_{2}>0$ such that

$$
K_{0}(x)=4 \prod_{j=1}^{k}\left|x-p_{j}\right|^{-2 n_{\jmath}} \prod_{j=1}^{l}\left|x-q_{j}\right|^{2 m_{\jmath}} \geqq|x|^{-2(N-M)}, \quad|x| \geqq r_{2} .
$$

Note that our condition means that $N-M \leqq 1$. 
We next restrict ourselves in (83) to the region $|x|=r \geqq r_{0}=\max \left\{r_{1}, r_{2}\right\}$, where $r_{1}$ and $r_{2}$ are determined respectively by (79) and (84). In view of (76), (77), (79), and (84) we have

$$
h(x) \geqq \frac{1}{1+C\left(r_{0}\right)}|x|^{-2(N-M)} e^{\prime \prime}, \quad|x| \geqq r_{0}
$$

Consequently we derive from (83) and (85) the inequality

$$
\frac{1}{r}\left(r W_{1}\right)_{1} \geqq \frac{C}{r^{2(N-M)}} e^{W}, \quad r \geqq r_{0},
$$

where we have used the Jensen inequality

$$
\frac{1}{2 \pi} \int_{0}^{2 \pi} e^{w} d \theta \geqq \exp \left(\frac{1}{2 \pi} \int_{0}^{2 \pi} w d \theta\right)=e^{W}
$$

To study (86) it is convenient to introduce the new variables $t=\ln r, \sigma(t)=$ $W\left(e^{t}\right)=W(r)$, and the initial point $t_{0}=\ln r_{0}$. Then (86) becomes

$$
\sigma_{t t} \geqq C e^{2(1-[N-M]) t+\sigma} \geqq C_{0} e^{\sigma}, \quad t \geqq t_{0}, \quad C_{0} \equiv C e^{2(1-[N-M]) t_{0}}
$$

because $1-[N-M] \geqq 0$ Here $\sigma_{t}=d \sigma / d t$

Recall that in (76) the function $h$ is positive except at $x=q_{1}, q_{2}, \quad, q_{l}$ Hence $r W_{1}(r)>0$ for all $r>0$. Returning to the variables $t, \sigma$ we have $\sigma_{t}(t)>0$ for all $t$.

Multiplying (87) by $\sigma_{t}>0$ and integrating we have

$$
\sigma_{t}^{2}(t)-\sigma_{t}^{2}\left(t_{0}\right) \geqq C_{0}\left(e^{\sigma(t)}-e^{\sigma\left(t_{0}\right)}\right)
$$

Using the fact $\sigma_{t}>0$ again and setting $\eta_{0}=\sigma_{t}^{2}\left(t_{0}\right)-C_{0} e^{\sigma\left(t_{0}\right)}$ we obtain then

$$
\sigma_{t}(t) \geqq \sqrt{\eta_{0}+C_{0} e^{\sigma(t)}}, \quad t \geqq t_{0},
$$

or

$$
\int_{\sigma\left(t_{0}\right)}^{\sigma(t)} \frac{d \sigma}{\sqrt{\eta_{0}+C_{0} e^{\sigma}}} \geqq t-t_{0}, \quad t \geqq t_{0}
$$

However the left-hand side of (88) has an upper bound since

$$
\int_{\sigma\left(t_{0}\right)}^{\infty} \frac{d \sigma}{\sqrt{\eta_{0}+C_{0} e^{\sigma}}}<\infty
$$

Thus (82) cannot be valid for all $t \geqq t_{0}$ This contradiction shows that our assumption that (8) has a finite-energy solution with $N \leqq M+1$ must be false.

For a solution $(\phi, \mathbf{A})$ of $(8)$ with $\operatorname{deg}(\phi)=N$ the transformation (17) gives us (22) and (23) with $N=\sum n_{j}$ (see [1,2] and the study in the last section). Thus by (26) again we conclude that $N>M+1, M \geqq 0$ In particular there is no degree one solution whatsoever.

To conclude this paper we make a few remarks

The nonexistence theorem for degree one solution is quite interesting Note that such a result is only proven for the self-dual system (8) and it is not known whether the same conclusion holds for the Euler-Lagrange equations of the model Indeed it 
is not clear whether the energy functional (3) permits the existence of any critical points whose energies are greater than those given by formula (13). The absence of degree one solutions of (8) already indicates that (13) is never attained at $N=1$. Thus if one could prove that the energy (3) has a minimizer, or a critical point, among all degree one field configurations it would mean that the system allows nonself-dual solutions as $S U(2)$ instantons [15]. At this moment the question in either direction is open. A comparable situation is the Abelian Higgs self-gravitating strings on $S^{2}$. There we know that a nonexistence result holds for $N=1$ solutions [16]. For the $O(3)$ sigma model here a possible approach is to first try to obtain radially symmetric "hedgehog" solutions as minimizers or critical points of (3) directly.

It is not clear whether the result on the number of parameters of the self-dual solutions for the case $N \geqq 2$ stated in Theorem 2 is optimal, although it seems to be exhaustive. As pointed out by Schroers in [3] one could hope to obtain more information by using index theorems to calculate the dimensions of fluctuations around a degree $N$ solution, as was done, for example, for the Abelian Higgs vortices [17].

The result on fractional values of the magnetic flux stated in Theorem 3 says that the parameter $\alpha$ is confined in the range (12). The interesting fact is that a larger $N$ (or $M$ ) leads immediately to a larger (or smaller) range of the flux $\Phi$ given in (14). Thus, magnetically, we could imagine that the points $q$ 's in $\phi^{-1}(\mathbf{n})$ counter-balance the points $p$ 's in $\phi^{-1}(\mathbf{s})$.

Note Added I am indebted to Haïm Brezis who showed me that the technical assumption (35) is, in fact, more or less, necessary and informed me that a class of equations of a related flavor to (26) appear as well in the Thomas-Fermi model in $\mathbf{R}^{3}$ (see Lemma 3 in Brezis [18]). Note that (26) also contains a sector for which the existence fails.

To see why (35) is almost necessary, it is most convenient to look at Eq. (28).

Recall that the finite-energy condition stated in Sect. 1 implies that

$$
V(x) \rightarrow-\infty \quad \text { as }|x| \rightarrow \infty
$$

and

$$
\int \frac{4 e^{-v_{1}+v_{2}+V}}{1+e^{-v_{1}+v_{2}+V}} \equiv \gamma<\infty .
$$

Define now $H(x)=$ the left-hand side of (28) and introduce the Newton potential

$$
W(x)=\frac{1}{2 \pi} \int(\ln |x-y|-\ln |y|) H(y) d y .
$$

We first show that $V=W+c$, where $c$ is a suitable constant.

In fact we already have $\Delta(V-W)=0$ in $\mathbf{R}^{2}$. On the other hand, by (89), we may assume $V<0$ everywhere (otherwise we can shift $V$ in $\Delta V=H$ by a large negative constant). Thus the entire harmonic function $h=V-W$ satisfies $h(x)<-W(x) \leqq C(\ln |x|+1)$. So a Liouville theorem type argument shows that $h$ must be a constant.

Therefore, using (90) and (91), we find directly that

$$
\lim _{|x| \rightarrow \infty} \frac{V(x)}{\ln |x|}=\lim _{|x| \rightarrow \infty} \frac{W(x)}{\ln |x|}=\frac{1}{2 \pi} \int H(x)=\frac{\gamma}{2 \pi}-2(N-M) \equiv-\beta,
$$


where we have also used (29). Inserting (92) into the condition (90) we obtain $\beta \geqq 2$ (if $\beta<2$, then $V(x)>-2 \ln |x|$ for $|x|$ large, which would imply the divergence of the integral (90)). Applying $\gamma>0$ in (92) we get $\beta<2(N-M)$. Consequently (35) is recovered

Acknowledgements I am grateful to D H Tchrakian for introducing me to the problems studied here, and to B J Schroers, for sending me his work [3] prior to publication and for stimulating communications I would also like to thank the anonymous referee for several helpful suggestions

\section{References}

1 Belavin, A A, Polyakov, A M : Metastable states of two-dimensional isotropic ferromagnets JETP Lett 22, 245-247 (1975)

2 Rajaraman, R: Solitons and Instantons Amsterdam: North Holland, 1982

3 Schroers, B J : Bogomol'nyi solitons in a gauged $O(3)$ sigma model Phys Lett B 356, 291-296 (1995)

4 Piette, B, Zakrzewski, W J : Skyrmion model in $2+1$ dimensions with soliton bound states Nucl Phys B 393, 65-78 (1993)

5 Nielsen, H, Olesen, P : Vortex-line models for dual strings Nucl Phys B 61, 45-61 (1973)

6 Jackiw, R, Lee, K, Weinberg, EJ : Self-dual Chern-Simons solitons Phys Rev D 42, 3488-3499 (1990)

7 Jaffe, A, Taubes, C H : Vortices and Monopoles Boston: Birkhäuser, 1980

8 Spruck, J, Yang, Y : The existence of non-topological solitons in the self-dual Chern-Simons theory Commun Math Phys 149, 361-376 (1992)

9 Chen, X, Hastings, S, McLeod, J B, Yang, Y : A nonlinear elliptic equation arising from gauge field theory and cosmology Proc R Soc London A 446, 453-478 (1994)

10 McOwen, $\mathrm{R}$ : Conformal metrics in $\mathbf{R}^{2}$ with prescribed Gaussian curvature and positive total curvature Indiana U Math J 34, 97-104 (1984)

11 McOwen, $\mathrm{R}$ : On the equation $\Delta u+K e^{2 u}=f$ and prescribed negative curvature in $\mathbf{R}^{2}$ J Math Anal Appl 103, 365-370 (1984)

$12 \mathrm{Ni}, \mathrm{W}-\mathrm{M}$ : On the elliptic equation $\Delta u+K(x) e^{2 u}=0$ and conformal metrics with prescribed Gaussian curvatures Invent Math 66, 343-352 (1982)

$13 \mathrm{Ni}, \mathrm{W}-\mathrm{M}$ : On the elliptic equation $\Delta u+K(x) u^{(n+2) /(n-2)}=0$, its generalizations, and applications in geometry Indiana U Math J 31, 493-529 (1982)

14 Sattinger, D H : Conformal metrics in $\mathbf{R}^{2}$ with prescribed curvature Indiana U Math $\mathrm{J} \mathbf{2 2}$, $1-4(1972)$

15 Sibner, L M, Sibner, R J, Uhlenbeck, K: Solutions to Yang-Mills equations that are not self-dual Proc Nat Acad Sci USA 86, 8610-8613 (1989)

16 Yang, Y : Cosmic strings on $S^{2}$ and criticality Proc R Soc London A, to appear

17 Weinberg, E J : Multivortex solutions of the Ginzburg-Landau equations Phys Rev D 19, 3008-3012 (1979)

18 Brezis, H : Some variational problems of the Thomas-Fermi type In: Variational Inequalities, Cottle, Gianessi and Lions, (eds ) New York: Wiley, 1980, pp 53-73 